\title{
BRAF Mutation Analysis Was Not Performed
}

National Cancer Institute

\section{Source}

National Cancer Institute. BRAF Mutation Analysis Was Not Performed. NCI Thesaurus.

Code C160332.

An indication that BRAF mutation analysis was not performed during the study. 